\title{
DÜBLIN
}

Technological University Dublin

ARROW@TU Dublin

\section{Impact of Loss of Generation on Bus Voltage Frequency and RoCoF}

\author{
Damien Doheny \\ Technological University Dublin, damien.doheny@tudublin.ie \\ Michael Conlon \\ Technological University Dublin, michael.conlon@tudublin.ie
}

Follow this and additional works at: https://arrow.tudublin.ie/engscheleart

Part of the Electrical and Computer Engineering Commons

\section{Recommended Citation}

D. Doheny and M. Conlon, "Impact of Loss of Generation on Bus Voltage Frequency and RoCoF," 2019 54th International Universities Power Engineering Conference (UPEC), 2019, pp. 1-6, doi: 10.1109/ UPEC.2019.8893605.

This Conference Paper is brought to you for free and open access by the School of Electrical and Electronic Engineering at ARROW@TU Dublin. It has been accepted for inclusion in Conference papers by an authorized administrator of ARROW@TU Dublin. For more information, please contact arrow.admin@tudublin.ie, aisling.coyne@tudublin.ie,gerard.connolly@tudublin.ie.

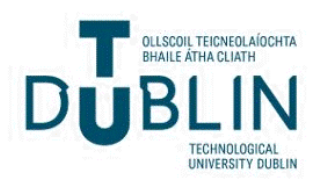




\section{Impact of Loss of Generation on Bus Voltage Frequency and RoCoF}

\author{
Damien Doheny \\ School of Electrical and \\ Electronic Engineering \\ Technological University Dublin, Ireland \\ Email: damien.doheny@mydit.ie
}

\author{
Prof. Michael Conlon \\ School of Electrical and \\ Electronic Engineering \\ Technological University Dublin, Ireland \\ Email: michael.conlon@dit.ie
}

\begin{abstract}
This study investigates how the loss of a generator impacts bus voltage RoCoF post-disturbance. The results show that the fastest bus voltage RoCoFs will occur close to generators impacted the most by the disturbance but this may not be the closest generator to the disturbance. The study also demonstrates a simple method that can be used to identify the generator that will be impacted most after a disturbance and hence the network location that will experience the fastest RoCoF.
\end{abstract}

Index Terms-Centre of Inertia, Bus Voltage Frequency, Bus Voltage RoCoF.

\section{INTRODUCTION}

Renewable energy targets have resulted in high penetration of non-synchronous generation. This is reducing power system inertia and could lead to power system frequency instability following a disturbance, such as the loss of generation [1]. Following such an event, an imbalance will exist between the generator mechanical power and the electrical load power. This imbalance will lead to the release of kinetic energy from the rotational mass of the generators, resulting in a reduction in their rotational speed and system frequency.

Systems with lower inertia have less stored rotational energy, hence, will experience a faster rate of change of frequency $(\mathrm{RoCoF})$ for a given power imbalance. If the power balance is not restored in an adequate time-frame, through primary frequency control, the system frequency could fall outside its safe operational limits, leading to the automatic disconnection of generators [2] and system instability. Therefore, primary frequency control in low inertia systems has a smaller time-frame in which to respond and stabilise the system frequency. As the time-frames get smaller we must increase the level of detail that we study the post-disturbance behaviour.

Fast RoCoF also poses other problems for power systems, such as tripping of generation due to gas turbine combustor lean blow-out [3] and mal-operation of distributed generation anti-islanding RoCoF relays [4]. Many solutions have been proposed to reduce fast RoCoF after a disturbance [5]. Some studies suggest synthetic inertia as a possible solution [6]-[8]. Synthetic inertia injects active power into the power system if the frequency deviates from a given value [8].

However, challenges exist to the optimum performance of synthetic inertia devices, such as time delays in RoCoF mea- surement and communications [5]. To improve the accuracy and speed of detection of RoCoF events, the behaviour of electrical power systems post-disturbance needs to be understood. This study investigates how the loss of a single generator impacts the magnitude of the bus voltage RoCoF and its location relative to the disturbance. The research shows, that for a given contingency, it is possible to identify the location of the largest initial RoCoF in the network.

\section{Power System Frequency}

The frequency of a power system is dependant on the rotational speed of the synchronous generators. In a multimachine power system the generators are synchronized to a common system frequency, but depending on the number of poles in the individual machines, the mechanical speed of the generators could be different. Therefore, it is convenient to express the speed of the generators in terms of their electrical speed, i.e. the frequency of the induced emf into the stator windings, rather than the actual mechanical speed of the machines.

In steady state conditions the electrical speed of all the generators are equal and constant. Under these conditions, the measured bus voltage frequency throughout the system will equal the electrical speed of the generators. Post-disturbance, the electrical speed of the generators will oscillate around a common centre frequency, called the centre of inertia, until the system settles to a new steady state. During this transient state the electrical speed of the generators will no longer be equal. The deviation in speed between the machines, and the oscillating power through the network, will result in local variations between all the bus voltage frequencies. Therefore, in transient state, three different frequencies needs to be considered;

1) Centre of inertia frequency

2) Speed of individual generators

3) Bus voltage frequency

\section{A. Frequency of Centre of Inertia}

Following the initial impact of a disturbance, all the machine speeds will oscillate around a common centre frequency. Depending on the nature of the disturbance and the resulting power imbalance, the centre of inertia will either increase or 
decrease at a rate proportional to the power imbalance and inversely proportional to the remaining system kinetic energy. The centre of inertia frequency as defined in [9] has an angular velocity $\bar{\omega}$ and post-disturbance mean acceleration $\frac{d \bar{\omega}_{\Delta}}{d t}$ given by;

$$
\begin{gathered}
\bar{\omega}=\frac{\sum_{i=1}^{n} \omega_{i} H_{i}}{\sum_{i=1}^{n} H_{i}} \\
\frac{d \bar{\omega}_{\Delta}}{d t}=-\frac{P_{\Delta}}{2 \sum_{i=1}^{n} H_{i}}
\end{gathered}
$$

where $P_{\Delta}$ is the power imbalance due to the disturbance, $H_{i}$ and $\omega_{i}$ are the inertia constant and angular velocity of machine $i$ respectively and $n$ is the total number of machines in the power system.

\section{B. Speed of Individual Generators}

At the instant of a disturbance the remaining machines will instantly respond to pick up the lost power. Some machines will pick up a greater share of the lost power compared to others. The response of each machine is determined by its share of the total synchronizing power to the location of the disturbance. This is a measure of the electrical distance from each machine to the disturbance. Closer machines will respond to a greater extent compared to more remote machines. The speed of the individual machines will begin to change in proportion to their response and inversely proportional to their individual inertias. Electrically closer machines with low inertia will experience a relatively fast rate of change of rotor speed (RoCoRS) and machines electrically far away with large inertia will experience a relatively slow RoCoRS. The synchronizing power coefficient is given by;

$$
P_{s i j}=E_{i} E_{j}\left(B_{i j} \cos \delta_{i j 0}+G_{i j} \sin \delta_{i j 0}\right)
$$

where $P_{s}$ is the synchronizing power coefficient between machines $i$ and $j$ and gives the change in electrical power of machine $i$ due to a change in angle between machines $i$ and $j$ with all other angles held constant. Its unit is pu power per radian [9], where $E_{i}, E_{j}, \delta_{i j 0}$ are the internal emfs and initial angle difference of generators $i$ and $j$, and $G_{i j}$ and $B_{i j}$ are the conductance and susceptance of the reduced network admittance matrix connecting the internal nodes of the synchronous generators $i$ and $j$.

The power response $P_{i \Delta}\left(0^{+}\right)$of each machine at the instant of the disturbance is given by [9];

$$
P_{i \Delta}\left(0^{+}\right)=\frac{P_{s i k}}{\sum_{\substack{j=1 \\ j \neq k}}^{n} P_{s j k}} P_{d \Delta}\left(0^{+}\right)
$$

For a system with $n$ generators, $P_{s i k}$ is the pre-disturbance synchronizing power between machine $i$ and the disturbance machine/bus $k$ and $P_{d \Delta}\left(0^{+}\right)$is the change in power at the instant of the disturbance, $t=0^{+}$. The initial RoCoRS of the individual generators is dependant on the inertia at each machine and also on the initial response of each machine. The initial RoCoRS, at the instant of the disturbance, of machine $i$ can be estimated by;

$$
\frac{d n_{i}}{d t}\left(0^{+}\right)=-\frac{P_{i \Delta}\left(0^{+}\right) f_{b}}{2 H_{i} S_{i}}
$$

It is important to note that (5) does not take into account bus voltage changes after the disturbance and this can also affect how each generator responds. Therefore (5) gives an estimate of the initial machine RoCoRS and is useful to determine which machine will be impacted the most after a disturbance.

\section{Bus Voltage Frequency}

In a power system it is not practical to measure and quantify frequency based on the centre of inertia. This would require real time information of the speed of all connected generators and and associated inertia [10]. It is more practical to measure and quantify frequency based on the rate of change of bus voltage angle. This can be achieved using phasor measurement units (PMUs) connected to buses throughout the system. However, the frequency measured by PMUs is not the frequency of the centre of inertia but the local bus voltage frequency and therefore, in a transient state, will not measure the same frequency as the centre of inertia.

The instantaneous voltage measured at a particular bus in a power system can be described by;

$$
v=V_{p k} \sin (\omega t+\phi)
$$

where $v$ is the instantaneous voltage, $V_{p k}$ is the peak voltage, $\omega$ is its angular frequency and $\phi$ is its phase relative to a reference. Its angular frequency $\omega$ is defined as the rate of change of the voltage angle;

$$
\omega=\frac{d \delta}{d t}
$$

Here the angular frequency $\omega$ is in units of radians per second and the instantaneous angle $\delta$ is in units of radians. Dividing (7) by $2 \pi$ gives the frequency $f$ in Hertz;

$$
f=\frac{1}{2 \pi} \frac{d \delta}{d t}
$$

The phase of the instantaneous voltage phasor continuously changes with time and after differentiating will give the frequency in radians per second. However, the phase of the rms voltage phasor does not change with time and after differentiating will result in a value of zero, and not the frequency of the voltage. To account for this, the change in voltage frequency $\Delta f$ can be calculated by considering the change in phase angle $\Delta \delta$ over a small time duration $\Delta t$. The change in frequency is then added to the base frequency $f_{b}$ to give the actual frequency. Equation (8) can be modified to reflect this;

$$
f=f_{b}+\frac{1}{2 \pi} \frac{\Delta \delta}{\Delta t}
$$


To confirm the accuracy of (9), a dynamic simulation of a disturbance was applied to the IEEE 39 bus system using Transient Security Assessment Tool (TSAT) [11]. The simulated voltage frequency was observed at one of the buses. Equation (9) was then applied to the same bus voltage angle. Figure 1 shows that the bus frequency calculated using the rate of change of the bus voltage angle is very similar to the bus frequency directly simulated by TSAT, confirming the accuracy of (9). The most noticeable difference is a large negative spike in the frequency at the instant immediately after the disturbance when the bus voltage frequency is calculated using the rate of change of voltage angle. This is due to the fact that there is an instantaneous change in the voltage angle at the instant of the disturbance, resulting in a negative infinite rate of change of bus voltage angle.

The large negative spike in frequency is not observed in the bus frequency directly from TSAT because a common method used by software for power systems simulation, is to calculate each bus frequency based on the numerical derivative of the bus voltage angle and filter the results using a washout filter [12]. The filtering action reduces the high frequency spike but does not completely remove it. It can be seen that there is still a trace of it present in the bus frequency calculated directly by TSAT.

Alternative methods to calculate bus frequency during simulations, without resulting in an initial high frequency spike, are proposed in [12] and [13]. However, disturbances on practical networks do produce very rapid changes in bus voltage angles [5], [14], [15] and this is what would be seen and measured by PMUs and other frequency measuring devices. Using alternative methods, such as that proposed in [12] and [13], to simulate bus frequency might mask a real world phenomenon that would not be observed in simulations.

Practical frequency measurement devices will use an algorithm to filter high frequency spikes but this introduces a delay into the measurement. Therefore the main constraints for filter parameters are to be insensitive to high frequency spikes but minimise the measurement delay [15]. The delay introduced by filtering needs to be minimised for synthetic inertia applications [15], so this implies that high frequency spikes could be observed more when measuring frequency and RoCoF for synthetic inertia applications. The nature of these transient frequencies needs to be understood better to improve frequency and RoCoF measurement.

1) Localised Frequency Variations: Equation (9) shows that a change in voltage angle will result in a change in bus voltage frequency. However, rapid changes in bus voltage angle may not be associated with a change in the speed of the synchronous generators as it could be the result of a transmission line switching events [14], [15]. Power oscillations between synchronous machines following a disturbance can also result in localised bus voltage frequency variations.

The transmitted power $P$ across a lossless transmission line with reactance $X_{l}$ and sending and receiving end voltages $V_{s}$ and $V_{r}$ with a phase difference of $\delta$ is given by; (a) Bus Voltage Frequency

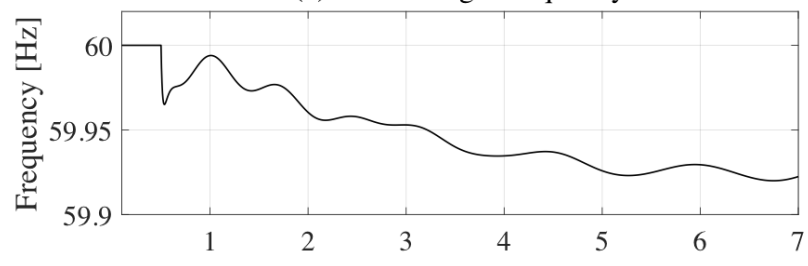

(b) Rate of Change of Bus Voltage Angle

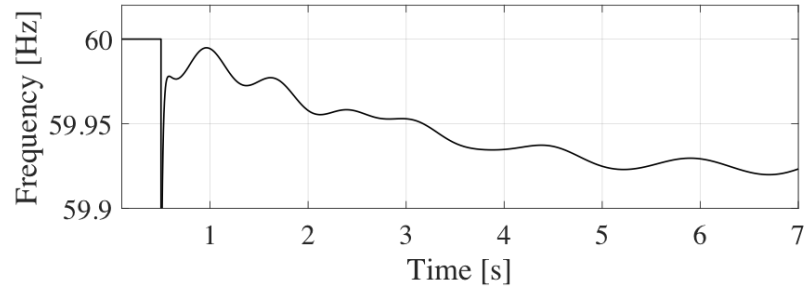

Fig. 1. Comparison between bus voltage frequency and frequency based on rate of change of bus voltage angle: (a) Bus voltage frequency directly from TSAT; (b) bus frequency based on rate of change of bus voltage angle.

$$
P=\frac{V_{s} V_{r}}{X_{l}} \sin \delta
$$

hence, the phase difference across the line is given by;

$$
\delta=\sin ^{-1} \frac{P X_{l}}{V_{s} V_{r}}
$$

Therefore, a change in power transmitted across the line will result in a change in phase angle of the receiving end voltage relative to the sending end voltage. Equation (9) shows that the bus voltage frequency is dependant on the change in phase angle. Hence, the change in receiving end phase angle will result in a frequency deviation relative to the sending end voltage.

After a disturbance the rotors of the synchronous machines will oscillate, resulting in power oscillations and local bus voltage frequency deviations throughout the network. Equation (11) shows that the phase difference across a transmission line is also dependant on the line reactance. Lines with smaller reactances will have smaller phase angle differences across them for a given power flow and therefore local bus frequency deviations will be smaller in strong networks with low impedance transmission lines.

\section{Methodology}

To investigate the behaviour of power system frequency immediately after a disturbance, a model of the IEEE 39 bus system was simulated using TSAT. A disturbance was applied to the system by disconnecting generator No. 1. The instantaneous bus frequency and RoCoF was recorded at each bus. Table I gives the parameters for the generators used in the simulation.

The RoCoF recorded at each bus was then compared to each bus location relative to the disturbance bus and also relative to the bus of the generator that was impacted to the greatest 
extent by the disturbance. The location was expressed in terms of the Thevenin impedance $Z_{i j}^{\text {thev }}$ between buses, given by [16];

$$
Z_{i j}^{\text {thev }}=Z_{i i}+Z_{j j}-Z_{i j}-Z_{j i}
$$

where $Z_{i i}$ and $Z_{j j}$ are the $\mathrm{i}^{\text {th }}$ and $\mathrm{j}^{\text {th }}$ diagonal elements, and $Z_{i j}$ and $Z_{j i}$ are the corresponding off-diagonal elements of the system impedance matrix.

The impact of the disturbance, in terms of RoCoRS, on the individual generators was estimated using (5) and this estimate was used to predict the generator that would be impacted the most and network location that would experience the fastest bus voltage RoCoF.

The same methodology was then applied to a more realistic system, a 1030 bus model of the all-island Irish transmission system.

TABLE I

GENERATOR POWER, RATING AND INERTIA

\begin{tabular}{|c|c|c|c|c|}
\hline $\begin{array}{c}\text { Machine } \\
\text { No. }\end{array}$ & $\begin{array}{c}\text { Generated } \\
\text { Power } \\
{[\mathrm{MW}]}\end{array}$ & MVA & $\mathrm{H}[\mathrm{s}]$ & $\begin{array}{c}\mathrm{H} \\
{[\mathrm{MWs}]}\end{array}$ \\
\hline 1 & 224 & 275 & 4.2 & 1155 \\
\hline 2 & 573 & 836 & 3.0 & 2533 \\
\hline 3 & 650 & 844 & 3.6 & 3020 \\
\hline 4 & 632 & 1175 & 2.9 & 3360 \\
\hline 5 & 508 & 1080 & 2.6 & 2809 \\
\hline 6 & 650 & 1086 & 3.5 & 3778 \\
\hline 7 & 560 & 1025 & 2.6 & 2707 \\
\hline 8 & 540 & 970 & 2.4 & 2358 \\
\hline 9 & 830 & 1684 & 3.5 & 5810 \\
\hline 10 & 750 & 1199 & 50.0 & 59950 \\
\hline
\end{tabular}

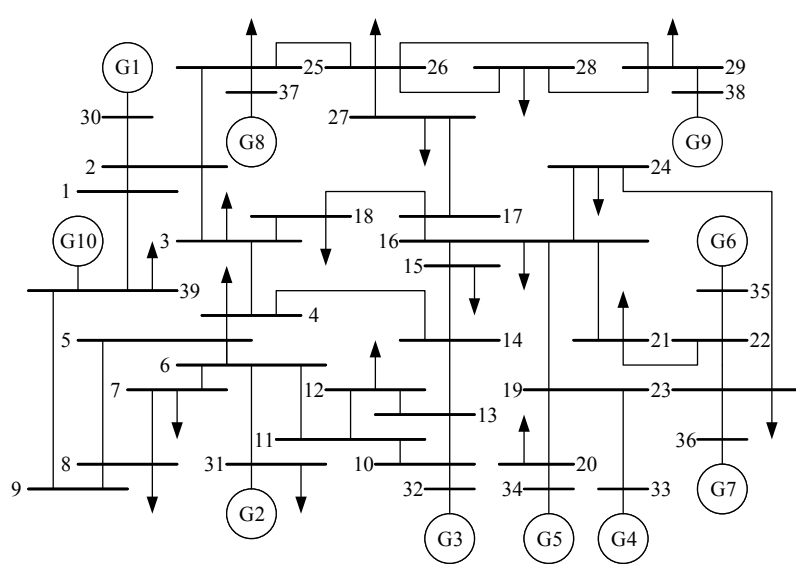

Fig. 2. IEEE 39 bus system

\section{Results And Discussion}

\section{A. IEEE 39 Bus System}

At the instant of the disturbance $224 \mathrm{MW}$ of generation was lost from the system. Table II shows how the remaining machines have been impacted after the loss of generator 1 . It can be seen that generator 8 has been impacted the most, as its initial RoCoRS is $-0.803 \mathrm{~Hz} / \mathrm{s}$. However, generator 10 is closest to the disturbance but only has an initial RoCoRS of $-0.04 \mathrm{~Hz} / \mathrm{s}$. Even thought generator 10 has $34 \%$ of the total pre-disturbance synchronising power to generator 1 , making it the electrically closest to the disturbance, generator 8 has been impacted the most because it has significantly less inertia. Therefore, generators electrically closest to the disturbance may not experience the greatest impact.

Table II also shows the predicted RoCoRS obtained using (5) and its error relative to the actual simulated RoCoRS. For some of the generators the predicted initial RoCoRS is very close to the simulated initial RoCoRS, however, there is a large error for others. What is noticeable is that generator 8 was predicted to have the fastest RoCoRS and the simulation results agree with this. This shows that it is possible to use (5) to identify the actual machine and hence area of the network that will experience the fastest RoCoF after a disturbance.

Figure 3 shows how the bus voltage RoCoF varies relative to the bus of the generator impacted the most by the disturbance, which is bus 37 . Figure 3 clearly shows a strong correlation between the magnitude of the instantaneous bus voltage Ro$\mathrm{CoF}$ and its location relative to the generator impacted the most after a disturbance.

These results show that when a disturbance occurs in a power system, faster instantaneous bus voltage RoCoFs will be observed closer to the buses of generators impacted the most by the disturbance but this might not be the closest bus or generator to the disturbance. It could be electrically far from the disturbance but close to the bus of a generator with low inertia.

Figures 4 and 5 show the simulated bus voltage frequency and centre of inertia frequency for the disturbance on the IEEE 39 bus system. It can be seen that the the frequency of the bus voltage oscillates about the centre of inertia. At any instant in time there can be a significant difference between any of the bus voltage frequencies, the speed of the machines and the centre of inertia frequency. The initial high frequency spike is also present and clearest on the bus frequency of the generator bus impacted most by the disturbance.

Figure 5a shows the simulated RoCoF at the centre of inertia, the bus voltage RoCoF at the bus of the most impacted generator and that generator's RoCoRS. Initial values of $-0.064 \mathrm{~Hz} / \mathrm{s},-2.67 \mathrm{~Hz} / \mathrm{s}$ and $-0.8 \mathrm{~Hz} / \mathrm{s}$ respectively were observed and this highlights the extreme differences that can exist between them.

Figure $5 \mathrm{~b}$ shows the centre of inertia and the filtered bus voltage $\mathrm{RoCoF}$ at the bus of the most impacted generator. Here, the bus voltage RoCoF is filtered using a $500 \mathrm{~ms}$ rolling window, as recommended in [15]. The initial spike in bus voltage RoCoF has been reduced to $-0.07 \mathrm{~Hz} / \mathrm{s}$, which is almost equal to the centre of inertia RoCoF. However, a significant delay has been introduced into the bus voltage RoCoF by this filtering action.

\section{B. 1030 Bus All-Island Irish System}

The EirGrid Group manages and operates the transmission grid across the island of Ireland [17]. They provided a 1030 
TABLE II

SYNCHRONIZING POWER, INITIAL RoCORS AND PREDICTED RoCoRS

\begin{tabular}{|c|c|c|c|c|}
\hline $\begin{array}{c}\text { Machine } \\
\text { No. }\end{array}$ & $\begin{array}{c}\text { Synch. } \\
\text { Power } \\
{[\%]}\end{array}$ & $\begin{array}{c}\text { Initial } \\
\text { RoCoRS } \\
{[\mathrm{Hz} / \mathrm{s}]}\end{array}$ & $\begin{array}{c}\text { Predicted } \\
\text { RoCoRS } \\
{[\mathrm{Hz} / \mathrm{s}]}\end{array}$ & $\begin{array}{c}\text { Error } \\
{[\%]}\end{array}$ \\
\hline 10 & 34 & -0.040 & -0.038 & 4.6 \\
\hline 8 & 21 & -0.803 & -0.587 & 26.9 \\
\hline 9 & 13 & -0.112 & -0.149 & -32.7 \\
\hline 6 & 8 & -0.072 & -0.142 & -96.1 \\
\hline 4 & 7 & -0.121 & -0.142 & -18.0 \\
\hline 3 & 6 & -0.122 & -0.127 & -3.6 \\
\hline 7 & 5 & -0.082 & -0.135 & -65.2 \\
\hline 2 & 4 & -0.053 & -0.114 & -113.7 \\
\hline 5 & 2 & -0.065 & -0.053 & 18.3 \\
\hline
\end{tabular}

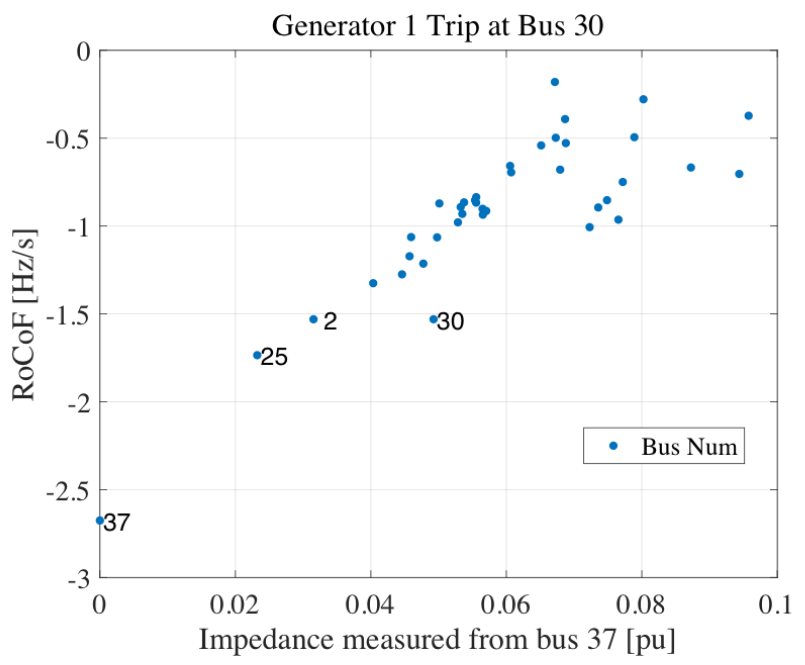

Fig. 3. Variation in instantaneous bus voltage RoCoF with distance from mos impacted generator on IEEE 39 bus system.

(a) Bus and Centre of Inertia Frequency

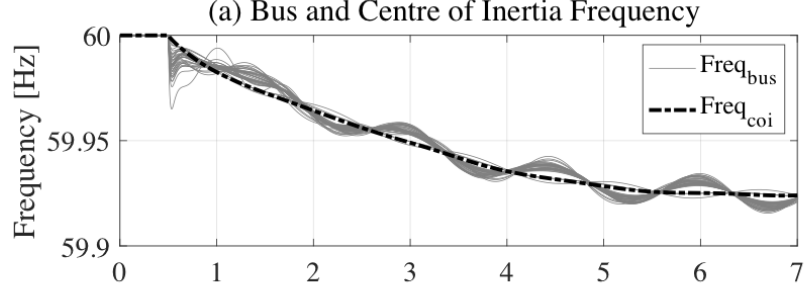

(b) Bus, Generator and Centre of Inertia Frequency

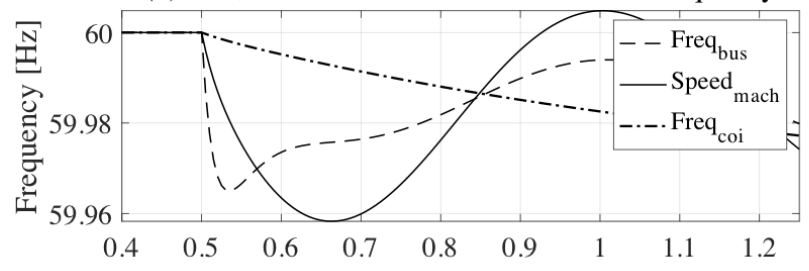

Fig. 4. Bus voltage frequency and centre of inertia frequency after the loss of generator No. 1 on the IEEE 39 bus system; (b) closer view of the speed of the generator No. 8, which is the generator most impacted, and its bus voltage frequency at bus 37 . (a) Bus, Generator and Centre of Inertia RoCoF

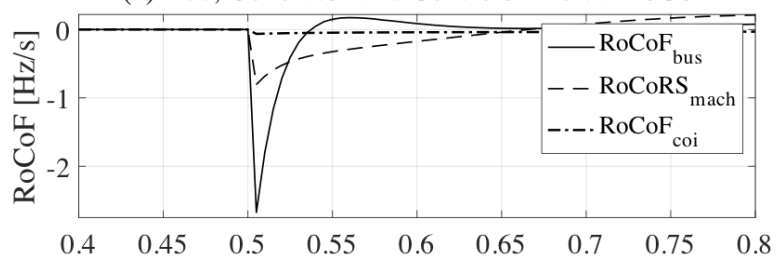

(b) Bus and Centre of Inertia RoCoF

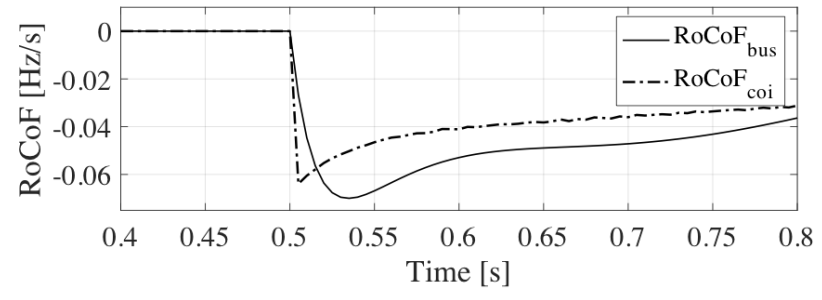

Fig. 5. (a) Generator No. 8, centre of inertia and bus No. 37 voltage RoCoF; (b) filtered bus voltage RoCoF and centre of inertia RoCoF.

bus model of the all-island Irish transmission system. Using this model a similar simulation was conducted as in part (A). Again a disturbance was simulated by tripping a generator. Figure 6 shows the immediate impact of the loss of generator 7 on the remaining machines. It can be seen that generator 12 is the electrically closest machine to the disturbance but is not impacted to the same extent as many other machines further from the disturbance. Generator 8 is one of the furthest machines from the disturbance but is impacted the most. This is because generator 8 has significantly low inertia and generator 12 has significantly high inertia. This result shows that generators far from a disturbance may be impacted disproportionally if they have significantly less inertia compared to the other generators and implies that buses close to generators with low inertia are more susceptible to fast RoCoF immediately after a disturbance.

Figure 7 shows the initial RoCoF recorded at the buses throughout the system and each buses' location relative to the bus of the generator impacted the most after the disturbance. It can be seen that the fastest RoCoF was observed at bus number 29971 and buses close to this bus. This is the bus of generator 8 , the machine that was impacted the most by the disturbance. It is intuitive to think that immediately after a disturbance the fastest bus voltage RoCoF will occur at or close to a disturbance, however, in this case, a machine electrically far from the disturbance was impacted the most because of its relatively low inertia and this resulted in the fastest bus voltage RoCoFs being observed electrically far from the disturbance. Equation (5) was used to identify generator 8 and bus 29971 as the network location that would experience the fastest RoCoF immediately after the disturbance

\section{CONCLUSION}

The results of the simulations show that immediately after the loss of a generator the fastest bus voltage RoCoFs will be seen at and close to the bus of the generator most impacted 


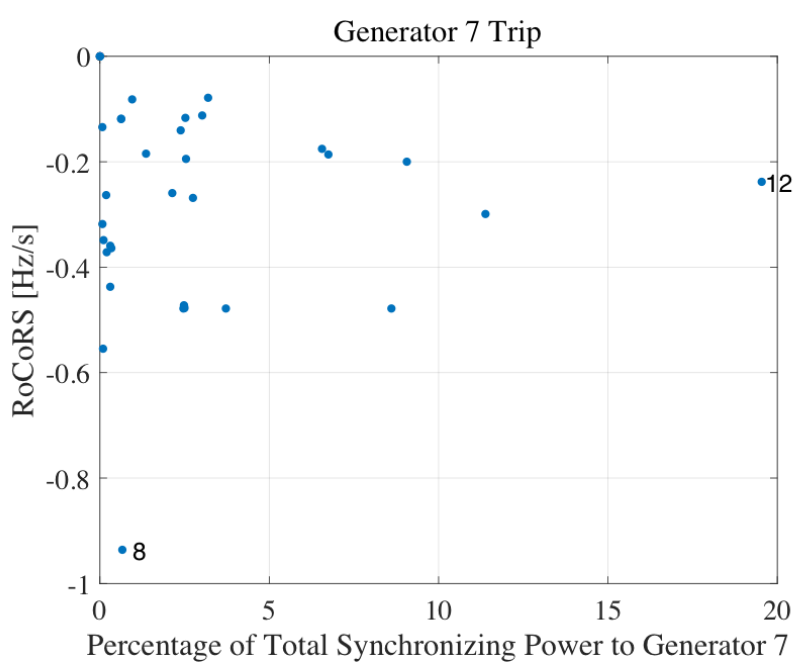

Fig. 6. Impact of loss of generator No. 7 on remaining machines compared to electrical distance from disturbance.

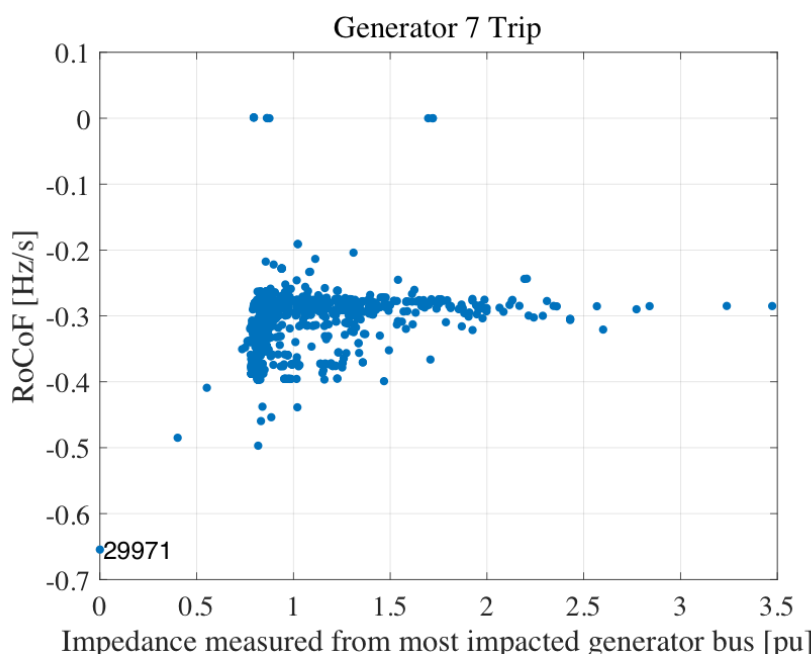

Fig. 7. Variation in instantaneous bus voltage RoCoF with distance from most impacted generator on Irish system.

by the disturbance. However, this may not be the electrically closest generator to the disturbance, it could be a generator far away but that has significantly lower inertia. The results also show that it is possible to identify the generator and hence network location of the fastest RoCoF for a given contingency using pre-contingency load flow data, share of machines individual synchronising power to the disturbance and local machine inertias.

This study highlights the differences between the centre of inertia and bus voltage RoCoF immediately after a disturbance. It shows that bus voltage RoCoF can be many times greater than the centre of inertia RoCoF, especially if the bus is close to a generator that has been impacted severely by the disturbance. It was seen that the magnitude of bus voltage RoCoF immediately after a disturbance is also dependant on its location in the network; buses closer to generators with low inertia are more susceptible to fast RoCoF. The effect of location on bus voltage RoCoF is not an issue if it is filtered because this will reduce the measured values, with the effect that all bus voltage RoCoF values will approximately be equal across the network. However, the action of filtering also introduces a delay into the measured bus RoCoF signal, so as time-frames to measure and trigger synthetic inertia become smaller, it may become necessary to consider how the location of a bus in a network affects its RoCoF immediately after a disturbance.

\section{ACKNOWLEDGMENTS}

The authors would like to thank EirGrid for their assistance.

\section{REFERENCES}

[1] P. Tielens and D. Van Hertem, "The relevance of inertia in power systems," Renewable and Sustainable Energy Reviews, vol. 55, pp. 999-1009, 2016. [Online]. Available: http://www.sciencedirect.com/ science/article/pii/S136403211501268X

[2] ENTSO-E, "Continental europe operation handbook - a1 load frequency control and performance," Report, 2004. [Online]. Available: https://docstore.entsoe.eu/fileadmin/user_upload/_library/publications/ entsoe/Operation_Handbook/Policy_1_Appendix\%20_final.pdf

[3] L. Meegahapola and D. Flynn, "Characterization of gas turbine lean blowout during frequency excursions in power networks," IEEE Transactions on Power Systems, vol. 30, no. 4, pp. 1877-1887, 2015.

[4] C. M. Affonso, W. Freitas, W. Xu, and L. C. P. d. Silva, "Performance of rocof relays for embedded generation applications," IEE Proceedings Generation, Transmission and Distribution, vol. 152, no. 1, pp. 109-114, 2005.

[5] W. Uijlings, "Rocof alternative solutions technology assessment," Report, 2015

[6] P. V. Brogan, R. J. Best, D. J. Morrow, K. McKinley, and M. L. Kubik, "Effect of bess response on frequency and rocof during underfrequency transients," IEEE Transactions on Power Systems, vol. 34, no. 1, pp. 575-583, 2019.

[7] S. Canevese, A. Iaria, and M. Rapizza, "Impact of fast primary regulation and synthetic inertia on grid frequency control," in 2017 IEEE PES Innovative Smart Grid Technologies Conference Europe (ISGT-Europe), Conference Proceedings, pp. 1-6.

[8] E. Spahic, D. Varma, G. Beck, G. Kuhn, and V. Hild, "Impact of reduced system inertia on stable power system operation and an overview of possible solutions," in 2016 IEEE Power and Energy Society General Meeting (PESGM), Conference Proceedings, pp. 1-5.

[9] P. M. Anderson and A. A. Fouad, Power System Control and Stability, 2nd ed. John Wiley \& Sons, 2003.

[10] ENTSO-E, "Future system inertia," Report. [Online]. Available: https://docstore.entsoe.eu/Documents/Publications/SOC/Nordic/ Nordic_report_Future_System_Inertia.pdf\#page54

[11] DSA Tools. (2019) TSAT. [Online]. Available: https://www.dsatools com/tsat/ [Accessed: 20/05/2019]

[12] F. Milano and A. Ortega, "Frequency divider," IEEE Transactions on Power Systems, vol. 32, pp. 1-1, 2016.

[13] G. Radman and M. Aghazadeh Tabrizi, "Calculation of power system dynamic frequency during simulation phase," International Journal of Emerging Electric Power Systems, vol. 13, 2012.

[14] T. Ackermann, T. Prevost, V. Vittal, A. J. Roscoe, J. Matevosyan, and N. Miller, "Paving the way: A future without inertia is closer than you think," IEEE Power and Energy Magazine, vol. 15, no. 6, pp. 61-69, 2017.

[15] ENTSO-E, "Frequency measurement requirements and usage," Report, 2018. [Online]. Available: https://docstore.entsoe.eu/Documents/SOC\%20documents/Regional_ Groups_Continental_Europe/2018/TF_Freq_Meas_v7.pdf

[16] P. Cuffe and A. Keane, "Visualizing the electrical structure of power systems," IEEE Systems Journal, vol. PP, pp. 1-12, 2015.

[17] EirGrid Group. (2015) Eirgrid group - about. [Online]. Available: http: //www.eirgridgroup.com/about/eirgrid-group/ [Accessed: 20/05/2019] 